\title{
humanidades
}

Revista humanidades

Enero-junio, 2016 •Volumen 6, número 1・ISSN 2215-3934・pp. 1-25

\section{El mito del trópico y la medicina en la experiencia colonial europea del África tropical}

DOI: http://dx.doi.org/10.15517/h.v6i1.24958

\section{Guillermo A. Navarro Alvarado}

Mestrando em Estudos Étnicos e Africanos. Posgrado em Estudos Étnicos e Africanos, Universidade Federal da Bahia, Brasil.

Correo electrónico: memo.naval.89@gmail.com

Todos los derechos reservados. Universidad de Costa Rica. Esta revista se encuentra licenciada con Creative Commons Reconocimiento-NoComercial-SinObraDerivada 3.0 Costa Rica.

Correo electrónico: humanidades@ucr.ac.cr / Sitio web: http://revistas.ucr.ac.cr/index.php/humanidades 


\title{
El mito del trópico y la medicina en la experiencia colonial europea del África tropical
}

\section{Resumen}

En el siguiente artículo se elabora un análisis descriptivo-relacional entre el mito del trópico y el nacimiento de la medicina tropical, a partir de la experiencia colonial europea del África tropical. Además, se abordan las condiciones socio-históricas de dicho proceso y las variantes míticas y pseudocientíficas que formaron parte de este.
Palabras clave: Trópico, medicina tropical, colonialismo, racismo, ideología.

\section{The Myth of the Tropic and the Medicine in European Colonial Experience of Tropical Africa}

\begin{abstract}
This article develops a relational-descriptive analysis between the myth of the tropic and the birth of the tropical medicine, framed in European colonial experience of Tropical Africa. In addition, they addressed the socio-historical conditions of this process and the mythical and
\end{abstract} Keywords: Tropic, tropical medicine, colonialism, racism, ideology. 
Durante la Edad Media, Europa parecía ser una fortaleza mental y material que se defendía imaginativamente de seres que comían carne humana; con cabezas similares a perros, seres compuestos o desordenados anatómicamente, vistos merodeando las orillas del continente, alejados de la "creación". Puede que suene descabellado, pero las tipologías de los monstra de la época lo mostraban de esta forma. Eran la representación de lo exterior, el marco interpretativo europeo que traducía las relaciones culturales entre distintos grupos sociales (Miles y Brown, 2003; Friedman, 2000).

De estas representaciones imaginativas, según (Miles y Brown, 2003), puede leerse una variante teórica que distinguirá los marcos interpretativos y de acción material de los europeos en relación con lo desconocido para ellos: el clima. Este factor marcará la larga interpretación, acción y experiencia que los europeos vivirán en los demás continentes. El clima fue un factor central en las explicaciones y acciones desarrolladas en o sobre las regiones exteriores a Europa; como dice Robert Miles y Malcolm Brown, se presentó como elemento diferenciador desde el inicio mismo de dichas tipologías medievales. "Within this tradition, a causal relationship between physical appearance, moral character and spatial location was asserted and, as in Greco-Roman thought, climate was considered to be a major determinant". [Dentro de esta tradición, la relación causal entre apariencia física, moral y localización geográfica era reconocida, y en el pensamiento Greco-Romano, el clima era considerado el mayor determinante] (2003, p. 24). 
Los tres elementos expuestos en esta cita, apariencia física, moral y localización geográfica, captan las tres líneas de diferenciación ideológica contrapuestas a la "cotidianidad europea", alimentados por las distintas realidades socio-institucionales de la Europa medieval. Estos elementos serán heredados y reproducidos a partir de categorías cada vez más empiristas, las cuales contendrán un concepto significativo para la comprensión de la exterioridad europea: el "trópico". 1

El clima será la pieza o núcleo central de la argumentación, el cual organizará a su alrededor distintos elementos y continuará estructurando nuevos mitos relacionados con el medio ambiente, la raza, las capacidades cognitivas, las capacidades económicas y las posiciones culturales, entre otros.

El trópico o los trópicos son categorías relacionadas con distintos elementos culturales, sociales, históricos, anatómicos y geográficos, su base parte de la denominación climática, pero su carga semántica referirá a la experiencia europea en el proceso de colonización y explotación de las regiones "exteriores". En estos términos, Trópico suele acoplarse a la exterioridad o a la periferia, parte de patrones etnocéntricos interpretativos que sedimentan el concepto con representaciones y experiencias ideológicas complejas, las cuales pueden ser interpretadas como proyecciones sociales, raciales, culturales o geográficas; es un concepto que acopló en sí fines expansivos y que condujo a la mistificación abstracta del espacio social colonizado. Así, trópico fue Argelia o el norte de África para los franceses, aunque su posición geográfico-climática se encontrara fuera de la zona intertropical (Curtin, 1989). 
Así pues, la categoría contendrá experiencias y explicaciones dirigidas a dirimir y resolver las contradicciones materiales que la empresa colonizadora encontraba, sedimentando y adjuntando a la categoría trópico nuevos significados. Tal proceso se significó en la estructuración de mitos relacionados con la necesidad "material" de la Colonia, que en términos dialécticos presentaban contradicciones materiales para los europeos, por así decir, problemáticas técnicas, como el tratamiento de los problemas de salud.

Este artículo analiza la relación de la categoría ideológica trópico, con la experiencia europea de las enfermedades en el África tropical y su mistificación. Se constituyen marcos explicativos de la impotencia y la desesperación colectiva del europeo.

Cabe señalar que esta posición se sitúa en el análisis de la percepción europea, como colonizadora y homogeneizadora, enmarcada en una tarea histórica contradictoria y heterogenia: la colonización. Por esto, el texto abarca la producción de mitos como categorías ideológicas suscritas al núcleo trópico, siendo reflejos de tales contradicciones, pero a su vez, encubriéndolas.

En este espacio marcado, la categoría trópico aparece como pieza fundamental para entender dos procesos centrales. Por un lado, los mitos sostenidos por la condición social del europeo en el África; por otro lado, el proceso social en el que surge y se instala la biomedicina como epistemología hegemónica en el tratamiento de las enfermedades.

Al unir tales categorías, surge un proceso poco explorado en el estudio de la medicina, el nacimiento de la Medicina tropical, como respuesta a las condiciones sociales coloniales, forma de acumulación de saber y 
línea fundadora de la institución médica moderna. Tales temas, suelen enfocarse como espacios ontológicos separados, distintos y desligados de la concepción del trópico en sus distintas dimensiones, tomándose solo la piedra angular el clima.

Este proceso es interesante, puesto que los estudios sobre medicina en África suelen contraponer dos sistemas públicos de salud, la biomedicina y la medicina "tradicional" africana; pero se disocian al plantear la primera como un grupo de prácticas cerradas y contrapuestas a la medicina "tradicional". En realidad, la biomedicina, debe comprenderse como un sistema, producto de procesos sociales de "acumulación", en un sentido abierto (Baronov, 2008).

¿Se podría pensar el descubrimiento de las bacterias o de los vectores de transmisión, sin el proceso colonial o la experiencia del trópico?, ¿qué tanto existe de invención o descubrimiento en procesos sociales de intercambio entre las diversas prácticas de salud africanas y las practicas occidentales?

Este campo queda abierto para una amplia exploración sistemática, la cual conduce no solo a pensar a la biomedicina como un "regalo" o un sistema meramente "racional" contrapuesto a una supuesta ignorancia o incapacidad de reacción de los habitantes de las zonas colonizadas.

Surge, entonces, la Medicina tropical como un conjunto de prácticas o campo epistemológico de especialización que experimenta, acumula y comprende, a partir de la idea del trópico. Además, reproduce mitologías y constituye un amplio proceso de acumulación de saberes, del que participan activamente las poblaciones llamadas "tropicales" y no solo los "occidentales". 
Actualmente, es común mirar el noticiero y encontrar noticias sobre nuevos virus encontrados en los trópicos, de los cuales, se dice, puede surgir la cura para el cáncer u originar algún exótico antídoto. No obstante, cuando se piensa en la institución clínica, la representación ideológica se dirige hacia la invención racional eurocéntrica. En ese sentido, Michel Foucault (2004) menciona dos fechas: 1658, con la clínica de La Bom; y 1720, con la escuela clínica de Edimburgo.

Así las cosas, el descubrimiento o la invención debe ser arqueologizada, en palabras de Foucault, además, deben tomarse en cuenta los procesos sociales de poder y acumulación que permitieron la constitución de la modernidad, la relación metrópoli-periferia, el sistema mundo capitalista, o la parte y el todo. Se podría afirmar que nada es completamente europeo, ni la propia experiencia. Esa sería, quizá, la tarea de la historia y de la medicina, una de las instituciones más modernas de la cotidianidad.

La noción de trópico tiene distintas acepciones y cargas en la experiencia colonial. La primera de estas tiene que ver con las condiciones climáticas: peligrosas, destructivas y dañinas para la salud. De esa forma, el clima se toma como piedra angular para comprender la diferencia, también para expresar la riqueza y la "distinción europea” como rasgo construido.

El trópico no es solo una meta-historia abstraída del conocimiento empírico, representado imaginativamente sin ningún tipo de prueba o experiencia; sino que la experiencia es su eje de construcción. Esta comienza desde la propia colonización de América, incluso antes, desde las propias expediciones y contactos del europeo con África (Curtin, 1990a).

Esta experiencia tomó una forma particular, la cual se entiende solo por la mediación de la enfermedad y la muerte tanto en el nativo, como en el colonizador. La muerte y la enfermedad son factores que construyen al

\section{El trópico africano y la condición europea}


clima como categoría común, posteriormente, desarrollarán la generalización del trópico. "To send officials to West Africa in the first decade of the twentieth century exposed the to a risk of death five to seven time higher than the expected death rate at home". [Enviar funcionarios a África Occidental en la primera década del siglo XX, era exponerlos al riesgo de muerte de entre cinco a siete por ciento más que la tasa de mortalidad esperada en casa]. (Curtin, 1989, p. 22).

En ese sentido, el trópico toma la carga histórica de la enfermedad y la muerte. Lo anterior se traduce, en primera instancia, por la supuesta reacción de los europeos a las condiciones climáticas, lo cual contrasta con el socializado mito de la exuberancia y riqueza de los trópicos, donde la "abundancia" era la regla (Curtin, 1990b). Estos dos puntos enmarcan al clima: la capacidad productiva e incapacidad del colonizador en la tarea de la supervivencia; se trata de polos contradictorios que caracterizan el significado de la categoría en un proceso social marcado por olas migratorias europeas y contrastes con las representaciones del trópico socializadas en Europa.

La condición social del europeo en este proceso estuvo marcada por profundas contradicciones que atañen a la posibilidad de muerte para el colonialista común, así como la necesidad de recursos para la manutención del proceso de acumulación. Con esto, podemos leer a la muerte, la enfermedad y el clima como los elementos claves para la comprensión de la mitologización del trópico.

Dentro de estas zonas, la costa occidental del África era una de las más peligrosas; sus condiciones climáticas y sus enfermedades provocaban numerosos esfuerzos investigativos y muertes, en una campaña que convirtió a la región en "cueva del hombre blanco", o más bien, su tumba, 
como bien lo refleja Curtin $(1989 ; 1990 a)$ en su detallado estudio sobre la mortalidad en el trópico ${ }^{2}$ :

"Tropical West Africa falls into a category all its own, with the highest morbidity and mortality rates for outsiders found any here in the world. The Sierra Leone rate of more than 400 per thousand was somewhat higher than usual, but peacetime rates of 100 to 200 per thousand had been common enough in the past and were to persist for several to come”. [E1 África Tropical Occidental cae en una categoría aparte, con las tasas más altas de morbilidad y mortalidad encontradas en cualquier parte del mundo (colonial) para los forasteros. La tasa de mortalidad en Sierra Leona de más de 400 por mil, era algo mayor de lo habitual, pero las tasas en tiempos de paz de 100 a 200 por mil, habían sido lo bastante común en el pasado e iban a persistir durante varias décadas] (1989, p. 18).

Este fenómeno experimentado por los europeos, principalmente en la región occidental de África, fue la base para la creación imaginativa y hermenéutica del clima tropical (Livingstone, 2002), sedimentando condiciones ideológicas con una noción de clima ampliada hacia una lectura racial.

El trópico africano se constituyó en un mito cargado de altas tasas de mortalidad para los europeos, lo que conllevó a sostenidos esfuerzos institucionales para mejorar la capacidad de respuesta del sistema de salud colonial, ante tales problemas. Estos esfuerzos se pueden sintetizar en cinco grandes grupos de acción: la construcción de infraestructura colonial (tomando en cuenta la altitud como factor), el manejo de las aguas residuales como un problema de planificación, el tratamiento del agua de 
consumo, la ventilación de las estructuras y la higiene personal como eje central (Curtin, 1989). La aplicación de dichas acciones no fue exclusivamente para el África tropical, sino que estas surgieron de sucesivas experimentaciones en distintas colonias y continentes, que en la singularidad de esta experiencia coincide con descubrimientos científicos específicos.

"The intensive colonization and territorial demarcation of tropical Africa occurred between 1880 and 1920, coincides with a period on human history marked by rapid changes in bioscientific theory". [La colonización intensiva y demarcación territorial del África tropical se produjeron entre 1880 y 1920, lo que coincide con un período en la historia humana marcada por los rápidos cambios en la teoría biocientífica] (Tilley, 2004, p. 23).

En esta práctica, el trópico se convirtió en un campo de experimentación científica para la solución de los problemas de salud europeos, en favor de los objetivos de colonización. Esto se demuestra por la tipología de disciplinas creadas durante el siglo XIX, topografía médica, ecología médica, botánica, medicina tropical, etc. (Tilley, 2004; Curtin, 1989).

El trópico, entonces, se convirtió para el imaginario europeo en un espacio patológico (Livingstone, 2002) y África tropical en el espacio más peligroso de todos. "Tropical Africa was the most dangerous place in the world for Europeans, but the number of European troops there was not enough to be used comparatively over a long period of time”. [El África tropical era el lugar más peligroso del mundo para los europeos, pero el número de tropas europeas no era el suficiente para ser utilizado comparativamente durante un largo período de tiempo] (Curtin, 1989, p. 17). 
Con esto, la percepción fenomenológica de la muerte en el África constituyó el mito central dentro del núcleo climático-tropical; el cual, al no poder compararse proporcionalmente con las demás colonias, alimentó el mito general de la incapacidad europea en la conquista del África.

No se puede dejar de lado que parte del modelo interpretativo climáticotropical fue alimentado por el paradigma médico preponderante hasta el siglo XIX, la teoría humoral, la cual constituyó la base de prácticas médicas que restringían la alimentación y constituían practicas básicas de la vida social en las colonias, como la higiene (Rodrigues, 2014; Curtin, 1989).

Esta constituía un modelo basado en el equilibrio de los fluidos supuestos como básicos para el ser humano, lo que calzaba con la interpretación del trópico como nocivo para el equilibrio europeo, por contraposición al equilibrio climático de Europa con sus cuatro estaciones.

De esa manera, se revelan dos antinomias: la del posicionamiento Trópico/Europa y desequilibrio/equilibrio. Ambas constituyen el fondo de la reproducción del mito y de su cambio de significación hacia la teoría racial del ambiente y la pertenencia genética.

Con esto la concepción del trópico comienza a dirigirse a dos líneas de interpretación y acción. La primera, exploración del ambiente como elemento productor de la enfermedad; y la segunda, una tipología racial que determina la resistencia "genética" a los espacios ambientales. Las dos concepciones se presentan como desarrollos que contienen las contradicciones y teorías planteadas, la cuales parecen haberse entremezclado en nociones y textos, dividiéndose en corpus o paradigmas separados para finales del siglo XIX. 
La condición del europeo y la noción de trópico tienen una base material: las enfermedades y la incapacidad material de respuesta de los europeos a estas. Lo anterior provocó la producción de mitos en torno al espacio ambiental y a las poblaciones autóctonas, así como una destrucción representacional de la respuesta autóctona a tales enfermedades. Es en el fenómeno etnocéntrico, aplicado ideológicamente, donde el mito del trópico encubrió la acumulación de saberes, como lo muestra Mark Harrison:

"Scientific ideas were seldom transplanted fully formed into colonial soil but were usually adapted and assimilated in significant ways. Moreover, much of what we think of as Western Science was produced in the colonies, rather than exported to them". [Las ideas científicas rara vez fueron trasplantadas completamente al suelo colonial, por lo general fueron adaptadas y asimiladas de manera significativa. Además, gran parte de lo que pensamos como la Ciencia Occidental, se produjo en las colonias, en lugar de exportarse a ellas] (Harrison, 2005, p. 60).

Como se planteó antes, la categoría de trópico contiene dos elementos contradictorios. El primero tiene que ver con la exuberancia/abundancia de estas regiones; y el segundo, con un ambiente hostil y difícil para los europeos. El primer elemento se traduce en una serie de enunciados ideológicos que justificaron la esclavitud, el trabajo forzado, el retraso cultural, la necesidad de la colonización y la exterminación. El segundo se relaciona con una serie de teorías explicativas y esfuerzos técnicomateriales, los cuales, en un contexto de altas tasas de mortalidad, se tradujeron en teorías de la higiene, saltos cualitativos en los tratamientos médicos y teorías raciales sobre tal fenómeno. 
"Many of these sixteenth-century generalizations lived on to influence the European theory of empire centuries later. One of the most influential was the set of beliefs sometimes called the myth of tropical exuberance. Its basis was the fact that the humid tropics and subtropics produced luxuriant vegetation all year round, with a wonderful variety of exotic fruits and valuable spices. The first impression, and one that often lasted, was that people living among such abundance had no need to work. They could simply enjoy what nature produced of her own accord. Sir Walter Raleigh, for example, reported of the Orenoqueponi of South America that "they never eat of anything that is set or sowen, and as at home they use neither planting nor manurance, so when they come abroad, they refuse to eat of aught, but that which nature without labour bringest forth”. [Muchas de estas generalizaciones del siglo XVI vivieron para influir siglos después a la Teoría Europea imperial. Unas de las más influyentes fue el conjunto de creencias algunas veces llamadas, mito de la exuberancia tropical. Su base era la presuposición de que las zonas tropicales húmedas y subtropicales producían una exuberante vegetación durante todo el año, con una maravillosa variedad de frutas exóticas y especies valiosas. La primera impresión, y una de las que más duró, fue que las personas (autóctonas) vivían entre tanta abundancia que no tenían necesidad de trabajar. Ellos simplemente disfrutaban de lo que la naturaleza produce por su propia voluntad. Sir Walter Raleigh, por ejemplo, informó que los Orenoqueponi de América del Sur "nunca comen nada preparado o cultivado, y como en casa no siembran ni trabajan la tierra, cuando viajan fuera de su tierra, se niegan a comer todo lo que no les haya dado la naturaleza sin trabajo] (Curtin, 1990, p. 132). 
En cuanto al segundo caso, la noción de clima ya ha sido trabajada, pero cabe insistir en que las tipologías imaginativas ya se presentaban en Europa desde la Edad Media, planteando una relación hipotética entre lo fenotípico y las condiciones ambientales. Sumado a esto, las respuestas dadas a tal diferenciación se encontraron en el marco ideológicoinstitucional religioso, el cual planteaba a la "creación" como europea, en el mito judío-cristiano, y a las demás diferencias como exterioridades del proyecto religioso (Miles y Brown, 2003).

Las preguntas que ocupaban a las autoridades colonialistas durante el siglo XIX se centraron en la incapacidad europea para la aclimatación. ¿Por qué el europeo no se puede aclimatar y ambientar al África tropical? Y, ¿qué hacer para que el europeo se ambiente a este espacio? Una vez aceptadas las nacientes disciplinas que relacionaban los elementos ambientales, geográficos y climatológicos con las razones de las tasas de mortalidad, se comenzaron a generar hipótesis socialmente sostenidas por las tipologías raciales históricas europeas, en donde la incapacidad de ambientación o aclimatación del europeo se tradujo a la búsqueda de razones raciales o fenotípicas. Estas, en lugar de mirar hacia las capacidades de las poblaciones autóctonas africanas para tratar las enfermedades, se centraron en el núcleo ideológico de la diferencia racial.

"This suggestion became one of the roots of pseudoscientific racism as it developed in the seventeenth and eighteenth centuries. Instead of being degraded humans, these overseas people might not be human at all-not, at least, in the same sense as Europeans were. The result came out in several different theories of polygenesis, or a separate creation for each of what we now recognize as the different varieties of humankind. The different human races were therefore 
separate and unequal by God's own intent in creating them differently. One of the most prominent of these views was Isaac La Peyrere's Prae-Adamitae (1655), holding that Adam and Eve were the last of a series of separate creations, and that non-Europeans were descended from the earlier and faultier acts". [Esta sugerencia se convirtió en una de las raíces del racismo pseudocientífico que se desarrolló en los siglos XVII y XVIII. En lugar de ser seres humanos degradados, los habitantes ultramarinos no eran humanos en absoluto, no, al menos, en el mismo sentido que los europeos eran. El resultado de esta idea devino en diferentes teorías sobre poligénesis, o una creación separada para eso que reconocemos como diferentes variedades o tipos de humanos. Las diferentes razas humanas eran, por tanto, separadas y desiguales por propia intención de Dios, al crearlas de manera diferente. Una de las publicaciones más destacadas dentro de estas visiones fue Prae-Adamitae de Isaac La Peryrere (1655), donde se sostiene que Adán y Eva fueron los últimos de una serie de creaciones separadas, y que los no europeos eran descendientes de los actos anteriores y fallidos] (Curtin, 1990b, p. 133).

Así pues, el retorno de los mitos exteriores de las gráficas medievales europeas y los marcos ideológicos religiosos, comenzaron a utilizarse como explicaciones institucionales de la incapacidad de aclimatación. "Early racism drew even stronger support from the evidence of differential mortality overseas". [El naciente racismo obtuvo un fuerte soporte de las evidencias sobre las diferentes tasas de mortalidad en las distintas zonas ultramarinas] (Curtin, 1990b, p. 133). 
Mircea Eliade (2000) plantea que el mito tiene la función de contar a los hombres el por qué de los eventos de hoy, de su actualidad, los eventos constituidos en su origen, como puntos ontológicos, el principio del todo por el cual ellos son como son. Se concuerda en lo anterior; sin embargo, es necesario aunar que estos cumplen una función social y que además son sostenidos por una estructura de poder que los socializa.

En ese sentido, el mito de la diferencia, de la creación, del principio como fuente (promovido por las instituciones sociales que crearon el mito europeo), son el principio a comprender. El trópico, como mito, tenía una función social: empujar a los europeos, de una Europa contradictoria, para que dejaran su tierra en busca de condiciones materiales básicas. No obstante, también debía explicar el porqué de las sucesivas muertes en espacios que se suponían salvajes o inferiores.

Así las cosas, el mito, en tanto recurso para resolver dicha contradicción, toma un nuevo giro y se convierte en un cuerpo explicativo que se sostiene en las ideas mitológicas europeas y en las nuevas formas de comprensión del medio ambiente. Lo anterior genera como resultado una serie de teorías e hipótesis que se irán afinando conforme la experiencia empírica avanza.

Ahora bien, los programas investigativos como la geografía, la botánica y la naciente biología, se entremezclan con las tradiciones ideológicoinstitucionales, recuperando la argumentación de la diferencia física y fenotípica como variables dependientes del medio ambiente; de esa manera, se respondió a la pregunta sobre la causa del fracaso ambiental del europeo en el África tropical. Por lo tanto, se argumentaba que el europeo se abstraía al ambiente físico-genético "normal", hacía uno acoplado a nativos "menos evolucionados" o "racialmente diferentes".

\section{Racismo, ciencia, ambiente}


David N. Livingston (1974, p. 76) plantea que este fenómeno se puede interpretar como una transición hermenéutica europea hacía sus nativos, sobre la noción de patología adjudicada al trópico; así como del núcleo ideológico clima, hacia la cultura. El europeo construyó una economía moral del trópico que se tradujo en jerarquías raciales.

Lo interesante de esta visión es que tal proceso parece haberse desarrollado durante el auge de la concepción climática y ambiental, lo que llevó a un buen sector de la "ciencia colonial" (Harrison, 2005) a nuevos avances que permitieron la comprensión de los vectores trasmisores de las fiebres, entre otros. Con esto se presenta un problema: ¿cómo es que la desviación racial no eliminó los esfuerzos teóricos ambientales si ambos marcos de interpretación del problema se entremezclaban?

"But as the possibility of White acclimatization on the tropics became more established, writers transferred tropical pathology form the natural environment to the native population". [Mientras la posibilidad de la aclimatación blanca (europea) en los trópicos se convirtió en algo estable y común, los escritores comenzaron a transferir las patologías tropicales del medio ambiente a las poblaciones nativas] (Livingstone, 2002, p. 75).

Una de las respuestas a esta contradicción recuerda la noción de Eliade, en la que el mito sigue alimentando la explicación, es una constante para la organización social que en el estado colonial toma un marco ideológico; es el vínculo estructural dinámico de las prácticas sociales y su expresión ideológica (Châtelet, 1968). La ciencia colonial promovía dos marcos científicos, uno para lograr mayor eficacia en la colonización y disminuir las muertes de los europeos (apropiándose también de conocimientos autóctonos sin reconocerlo) y otro para la comprobación racional instrumental del porqué de una tarea de tales dimensiones. 
Se puede plantear que el nacimiento de la medicina, y en especial de la medicina tropical, retoma como principio los dos programas, cuyo hermano fracasado, pero ideológicamente eficaz, vendría a ser el racismo científico. Así pues, la biomedicina y los marcos epistemológicos de comprensión de las enfermedades llamadas "tropicales", que también afectaban a las ciudades europeas en épocas de verano o variedades específicas, se entrenaron y se desarrollaron a la luz de este panorama mixto, no solo en los programas franceses del siglo XVII.

Queda manifiesto, entonces, que la medicina como disciplina nace dentro de un espacio social marcado por el racismo y la explotación, donde se exploran en favor de ciertos intereses, técnicas y epistemologías que conllevan historias complejas; que no son en sí un regalo orgánico brindado por la modernidad hacia lo "tradicional", sino un proceso de construcción contradictorio que se alimentó de un espacio plagado de poder direccionado y de acumulación mistificada.

En este espacio marcado, se define a la medicina tropical como el encuentro entre la investigación biológica y las ciencias médicas, en la búsqueda de curas para las enfermedades del trópico (Tilley, 2004). El África tropical, con sus altas tasas de mortalidad europea, se convirtió en el laboratorio por excelencia para este nuevo programa investigativo.

A primera vista, este se presenta como un marco investigativo cerrado, con objetivos precisos en relación con la observación de la naturaleza; sin embargo, esta disciplina o especialización médica nace de la necesidad del control médico para mejorar la salud de los europeos que colonizaban. 
Dicha disciplina suele aislarse de su espacio social; no obstante, para efectos de este trabajo, se dirá que esta se alimentó de las contradicciones materiales e ideológicas antes expuestas, retomando las tesis racistas europeas y la categoría de trópico como categoría ideológica. En un artículo publicado en el año 1905, titulado The Progress of Tropical Medicine, se pueden leer los argumentos ideológicos antes expuestos, como raíces discursivas y materiales de la naciente disciplina médica.

"On January 12th Mayor Ronald Ross delivered a lecture on the progress of tropical medicine at Liverpool before a large and distinguished audience. He Said that large part of the British Empire lay within or near the tropics -the East and West indies, the chain of West African Colonies, and many isolated possessions and protectorates, such as Egypt and British Central Africa. In many of these, and no less in many of the tropical possessions of France, Germany, Holland, and Belgium, disease was the principal bar to progress. Particularly in the case of tropical Africa one could not fail to be struck by the fact that that great continent still remained uncivilized, in spite of its proximity to Europe, much greater than that of Asia of America, which however, had advanced for more the Africa in the path of civilization. All things considered, he was convinced that the principal cause for this backwardness was the socalled unhealthiness of the African "climate", where, as used frequently to occur on the West Coast, half the Europeans in a settlement might be obliterated by some epidemic within a few weeks, and rapid progress was hardly possible. The ever-present endemic diseases of those vast areas, and of many more tracts of the tropical world, paralysed administration by direct mortality, and also by compelling a constant return to Europe in search of healthy; it paralysed commerce for the same reasons, vastly increased the 
cost and dangers of military expeditions, and checked the missionary and explorer on the threshold of the countries which they come to open up. Not only on the European visitors, but on the wretched nations them selves, did they wreak their evil. Before prosperity in such areas could even be dreamt of they must set themselves to the wheel of tropical sanitation and struggle against those maladies which were the cause of the evil. There was only one way, and that was to ascertain their nature. Mere empirical efforts to cure them or even to prevent them had in most causes failed for many centuries". [El 12 de enero el alcalde Ronald Ross dio una conferencia sobre el progreso de la medicina tropical en Liverpool, ante un público numeroso y distinguido. Dijo que gran parte del Imperio Británico estaba dentro o cerca de las zonas tropicales -las Indias Orientales y Occidentales, la cadena de colonias en el África Occidental, y muchas posesiones y protectorados aislados, como Egipto y el África central británico. En muchas de ellas, y no menos en muchas de las posesiones tropicales de Francia, Alemania, Holanda y Bélgica, la enfermedad era la principal barrera para el progreso. Particularmente en el caso del África tropical, uno no podía dejar de sorprenderse por el hecho de que ese gran continente aún permaneciera incivilizado, a pesar de su proximidad a Europa, mucho más que Asia y América, los cuales, habían obtenido mayores avances en el proceso civilizatorio que África. Ante todo lo considerado, estaba convencido de que la principal causa de este retraso era la llamada insalubridad del "clima" en el África, en el que, así como es utilizado para producir en la costa oeste, también la mitad de los europeos que trabajan en esta región, pueden morir en unas cuantas semanas por alguna epidemia, con lo que un rápido progreso era casi imposible. Las siempre presentes enfermedades 
endémicas de estas vastas zonas, y de muchas más extensiones del mundo tropical, paralizan administraciones por la mortalidad directa que estas provocan, lo que también obliga al constante retorno a Europa en busca de salud; esto paraliza el comercio por las mismas razones, aumenta enormemente el costo y peligro de las expediciones militares, y asusta a los misioneros y exploradores en el umbral de los países a los que vienen a abrir paso. No sólo los visitantes europeos, sino también los miserables nativos sufren esta maldad. Antes que la prosperidad sea soñada para estas zonas, deberían establecerse acciones de saneamiento tropical y lucha contra esos males que son en sí la causa de todo mal. Sólo hay un camino para eso, y ese es determinar su naturaleza. Más esfuerzos empíricos para curar los males o por lo menos, evitar que este caso falle también para los demás siglos] (1905, p. 156).

Este amplio extracto sintetiza las condiciones objetivas del nacimiento y desarrollo de la medicina tropical, la cual contenía los núcleos míticoideológicos reproducidos en las categorías de trópico y clima. Su génesis programática se empeñó en el alcance de objetivos durante la empresa colonial, alimentando las ideas de separación racial, las cuales, no obstante, materialmente constituyeron una lógica de separación y apropiación del saber de los nativos africanos.

Bajo este proceso socio-histórico, emergen las escuelas de medicina tropical, por ejemplo, London School of Tropical Medicine y Liverpool School of Tropical Medicine. Estas, como instituciones, obedecen a un objetivo específico: hacer habitable el trópico para el europeo. Esta visión se expresa muy bien en un artículo publicado el 20 de mayo de 1905 en el British Medical Journal, el cual recoge algunos objetivos y necesidades de 
la naciente disciplina en la búsqueda de financiación para la London School of Tropical Medicine.

"The response to appeal was sufficiently liberal to free the school from adopt of $£ 6,000$, and to place $£ 4,000$ to its credit. This is something, but it is not by any means all that is required to enable the school to fulfil its destiny as a nursery of scientific pioneers whose mission it is to make the tropics habitable by civilized men". [La respuesta al llamado fue suficientemente liberal para liberar a la escuela de la deuda de $£ 6,000$, y para colocar $£ 4,000$ en su crédito. Esto es algo, pero no es de ninguna manera todo lo que se requiere para que la escuela pueda cumplir su destino como un cuidador de pioneros científicos, cuya misión es hacer a los trópicos habitables por los hombres civilizados] (1905, p. 1105).

La medicina tropical reproduce el mito del trópico y forma parte de la ciencia, la cual en su desarrollo se sitúa en un espacio continuo de contradicciones; producto de intereses y relaciones de poder, que en este caso se significan como coloniales. Aunque a primera vista la medicina se presente como una institución cronológica, neutra y benevolente, lo cierto es que su producción se regía en el contexto colonial por intereses directos de acumulación y dominación. La medicina tropical sintetiza los procesos en nuevas versiones que darán resultados positivos para el avance colonial y el establecimiento de la epistemología médica en las metrópolis y las periferias, constituyendo en dicho proceso, una suerte de industria alimentada por las precarias condiciones de salud que los europeos vivían y expandían en los nativos. 
Amin, S. (2009). Eurocentrism. Modernity, Religion, and Democracy. A Critique of Eurocentrism and Culturalism. New York: Monthly Review Press.

Baronov, D. (2008). The African Transformation of Western Medicine and the Dynamics od Global Cultural Exchange. Philadelphia: Temple University Press.

Carmichael Low, G. (1935). Tropical Medicine. The British Medical Journal, 2, (3902), pp. 715-717.

Châtelet, F. (1968). Ideología y Verdad. En H. Lefebvre, y F. Châtelet, Ideología y Verdad (pp. 41-62). Buenos Aires: Carlos Pérez Editor.

Comaroff, J., y Comaroff, J. (1992). Ethnography and the Historical Imagination. Oxford: Westview Press.

Cooper, F. (2005). Colonialism in Question. Theory, Knowledge, History. Berkeley: University of California Press.

Curtin, P. D. (1989). Death by migration: Europe's encounter with the tropical world in the nineteenth century. New York: Cambridge University Press.

Curtin, P. D. (1990a). The End of the "White Man's Grave"? NineteenthCentury Mortality in West Africa. The Journal of Interdisciplinary History, 21, (1), pp. 63-88.

Curtin, P. D. (1990b). The Environment beyond Europe and the European Theory of Empire. Journal of World History, 1, (2), pp. 131-150.

Eliade, M. (2000). Mito e realidade. São Paulo: Perspectiva.

Elias, N. (1989). La soledad de los moribundos. México D.F: Fondo de Cultura Económica.

Foucault, M. (2004). El Nacimiento de la Clínica. Una arqueología de la mirada médica. Buenos aires: Siglo XXI.

Friedman, J. (2000). The Monstrous Races in Medieval Art and Thought. New York: Syracuse University Press. 
George, K. (1958). The Civilized West Looks at Primitive Africa: 14001800 a Study in Ethnocentrism. Isis, 49, (1), pp. 62-72.

Harrison, M. (2005). Science and the British Empire. Isis, 96, (1), pp. 5663.

Livingstone, D. N. (2002). Tropical hermeneutics and the climatic imagination. Geographische Zeitschrift, Bd. 90, H. 2, pp. 65-88.

Löwy, I. Historiography of Biomedicine,."Bio," "Medicine," and In Between. Isis, 102, (1), pp. 116-122.

Miles, R., y Brown, M. (2003). Racism. London: Routledge Taylor \& Francis Group.

Patterson, K. D. (1974). Disease and Medicine in African History: A Bibliographical Essay. History in Africa, 1, pp. 141-148.

Power, M., y Sidaway, J. D. (2004). The Degeneration of Tropical Geography. Annals of the Association of American Geographers, 94, (3), pp. 585-601.

Reed, A. C. (1929). Tropical Medicine. The Scientific Monthly, 29, (5), pp. 458-464.

Rodrigues, E. (2014). Discurso médico e práticas alimentares no Hospital Real de Moçambique no início do século XIX. História, Ciências, Saúde-Manguinhos 21, (2), pp. 609-627.

The Aims Of Tropical Medicine. (1905). The British Medical Journal, 1, (2316), pp. 1105-1106.

The Progress Of Tropical Medicine. (1905). The British Medical Journal, 1, (2299), pp. 156-157.

Tilley, H. (2004). Ecologies of Complexity: Tropical Environments, African Trypanosomiasis, and the Science of Disease Control in British Colonial Africa, 1900-1940. Osiris, 2nd Series, (19), Landscapes of Exposure: Knowledge and Illness in Modern Environments, pp. 21-38. 
El mito del trópico y la medicina en la experiencia colonial europea...

1. Esta reproducción puede ejemplificarse en las transiciones representacionales del "África Primitiva" en la mirada europea, donde los tres elementos juegan un papel organizativo. Tal proceso puede ilustrarse en los periodos expuestos en el sintético y clásico trabajo de Katherine George (1958).

2. Este es contrastado imaginativamente con el proceso vivido por el europeo en América, donde la tasa de mortalidad le favoreció en una primera etapa de colonización, teniendo esta un mayor impacto en las poblaciones autóctonas (Curtin, 1989).

Recibido: 03-agosto-2015

Aceptado: 19-noviembre-2016

Todos los derechos reservados. Universidad de Costa Rica. Esta revista se encuentra licenciada con Creative Commons Reconocimiento-NoComercialSinObraDerivada 3.0 Costa Rica. (CC BY-NC-SA 3.0 CR)

Correo electrónico: humanidades@ucr.ac.cr Sitio web: http://revistas.ucr.ac.cr/index.php/humanidades 